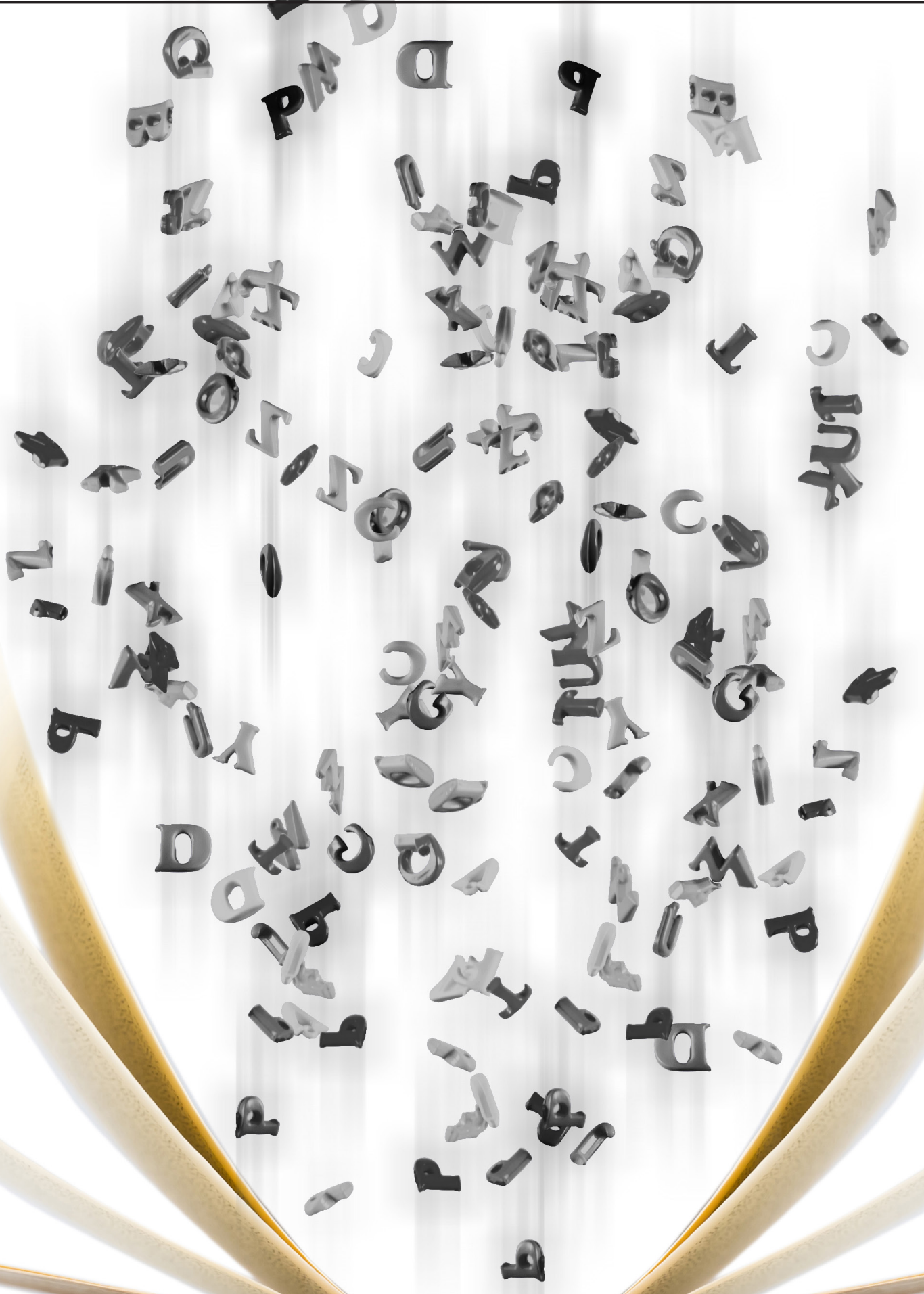




\title{
El Deleite de Enseñar y Aprender: Caso Fundación Bandera Blanca
}

\author{
Olga Marta Ramírez Hernández \\ Escuela de Diseño Publicitario \\ Universidad Fidélitas \\ San José, Costa Rica \\ oramirez80205@ufide.ac.cr
}

\begin{abstract}
This document contains a case study, in which the constructivist pedagogical model was applied at the Fundación Bandera Blanca, a non-governmental organization focused on children's care and protection. It was developed in the Project Management and Planning course (May to August 2019). The limitations of the organization to receive money donations in order to continue operating were studied. Course students created a website for the organization, successfully applying project-based learning and the constructivist model.
\end{abstract}

Key words: constructivism, non-governmental organizations.

\section{Introducción}

El desafío de enseñar en este milenio implica definir los conocimientos que requiere el estudiantado para que este "aprendizaje" sea realmente significativo y de impacto a la sociedad en general. Por lo tanto, la enseñanza debe lograr un equilibrio entre teoría y práctica para provocar empatía en el estudiantado. Los docentes deben visualizar nuevas perspectivas para enseñar e interactuar con los jóvenes, quienes han crecido con el auge del uso de las nuevas herramientas digitales lideradas por el uso de internet. Los docentes deben ser creativos y utilizar modelos pedagógicos que orienten la estimulación del aprendizaje en los jóvenes.

Muchos autores sostienen que el constructivismo es básicamente un enfoque epistemológico, basándose en la relación o interacción que se establece entre el objeto de conocimiento y el sujeto que aprende, es decir, la relación objeto-sujeto, para otros se trata de una nueva forma de conceptualizar el conocimiento (aprendizaje) [1].

El psicólogo suizo Jean Piaget, pensador y uno de los fundadores del constructivismo, afirmaba que "la fuente de las operaciones mentales no hay que buscarla en el lenguaje sino en la acción como base de la organización de la experiencia" [2]. Desde esa perspectiva la teoría debe buscar a la práctica y regresar a su vez, a un proceso de conceptualización y de asimilación. Esto con el fin de que ese conocimiento sea significativo. "Las acciones se transforman en esquemas que paulatinamente se desprenden de los modelos y los contextos y se interiorizan para dar lugar a las representaciones" [2]. En este modelo pedagógico el estudiante puede crear, aportar e innovar al aplicar conocimiento adquirido previamente. Se visualiza "al aprendizaje del sujeto como un proceso activo de construcción (o reconstrucción) del conocimiento y la labor del maestro como mediador entre el sujeto y el objeto en la apropiación de los conocimientos, es decir, de promotor de ese proceso interno [1].

Cárdenas [2] destaca que:

De acuerdo con Piaget (1973), el intelecto humano avanza desde lo sensoriomotor hasta lo conceptual lógico en la constitución de estructuras mentales; pero este proceso no se circunscribe a ninguno de los dos factores, pues el conocimiento no se manifiesta en etapas, sino que es integral, holístico, no obstante, la posibilidad de que, a veces, prevalezca uno sobre el otro, otros a través de manifestaciones más o menos caracterizadas e identificables en sus elementos.

De ahí, la importancia del contexto económico, político y social que permite la asimilación de conocimientos y a su vez, la puesta en práctica de ese "nuevo aprendizaje" por parte de los estudiantes. El docente se convierte en el facilitador de todo el proceso.

Para Mazarío [1] los principios fundamentales del constructivismo son:

1. La comprensión inicial de un objeto, proceso o fenómeno es local, no global. Las nuevas ideas son necesariamente introducidas y entendidas sólo en un contexto limitado.

2. El conocimiento no es recibido de forma pasiva, sino construido y reconstruido por el sujeto cognoscente de forma activa, interactuando con el objeto de estudio (relación objeto-sujeto).

3. La función cognoscitiva es adaptativa y permite al que aprende la construcción de explicaciones viables sobre 
sus experiencias, es decir, cuando un sujeto actúa sobre la información relacionándola con el conocimiento que ya posee, le imprime e impone así organización y significado a su experiencia.

4. El proceso de construcción de significados está siempre influenciado por el contexto histórico-cultural y económicosocial del cual el individuo forma parte.

5. Construir estructuras útiles de conocimiento requiere de una actividad esforzada e intencionada. El aprendizaje requiere una participación activa y reflexiva.

El modelo pedagógico del constructivismo contribuye a que el estudiantado pueda comprender, asimilar y asumir nuevos conocimientos y lo más importante que los pueda poner en práctica.

Este artículo tiene como objetivo presentar un caso de estudio, donde se aplicó el modelo pedagógico constructivista. El caso se realizó en el curso de Gestión y Planificación de Proyectos, de la Licenciatura en Diseño Publicitario con énfasis en Gestión de Proyectos. Para el desarrollo del caso se contó con el apoyo de la Fundación Bandera Blanca, una organización no gubernamental orientada al cuido y protección de la niñez. La aplicación del estudio fue de mayo a agosto del 2019.

La Fundación Bandera Blanca es una fundación ONG sin fines de lucro con un fuerte mensaje cristiano que busca ayudar a personas con problemas sociales enfocado en la comunidad infantil de San José.

La Fundación tiene dos locales. Una tienda en Barrio Luján y un comedor en Hatillo. También sirve como lugar para hacer Trabajo Comunal Universitario con estudiantes de universidades y voluntariado para extranjeros que quieran ayudar a la población con más dificultades.

Dentro de los proyectos de la Fundación tienen clases de inglés para niños, clases de arte, deportes, tutorías académicas entre otros

\section{a. Caracterización del problema}

La Fundación Bandera Blanca presenta dificultades en la comunicación con la que se dirige hacia distintos públicos de interés, los cuales se deben atacar de manera positiva para demostrar confianza, credibilidad y a su vez, lograr la maximización de los recursos que llegan a la Fundación mediante donaciones de dinero que brindan los públicos cautivos del extranjero.

La Fundación, a nivel de imagen corporativa, presenta debilidades de posicionamiento. Debido a que se creó un posicionamiento confuso en el público local costarricense que brinda apoyo y ayuda con el Trabajo Comunal Universitario. Cuando se habla de posicionamiento confuso, se refiere a cómo los distintos públicos perciben la Fundación Bandera Blanca, ya que estos la perciben como una organización que ayuda a la conservación del medio ambiente. Sin embargo, el objetivo principal de la Fundación es "ser una asociación de bien social sin fines de lucro, que emergió con la idea orientada de ayudar a instituciones, organizaciones, familias, niños y niñas que necesiten mejorar su calidad de vida, con la intención de manifestar sus inquietudes ante el panorama social, el bienestar de todos y busca la transformación siguiendo a Jesucristo, Señor y Salvador, llevando fe y esperanza a los necesitados, brindando alimentación $\mathrm{y}$ apoyo académico a poblaciones vulnerables para mejorar su calidad de vida" [3].

La Fundación no cuenta con un sitio web al idioma inglés como idioma universal, de fácil comprensión para quienes llegan a realizar voluntariado desde otros lugares del mundo y así estos puedan comprender las distintas áreas en las cuales puedan desarrollar y desempeñar sus habilidades y cumplir con la cantidad de horas de trabajo comunal universitario.

Otra de las dificultades que se le presenta a la Fundación es el lugar donde se encuentra el comedor, este está cubierto por unas rejas y no se encuentra con la respectiva rotulación, lo cual esto se les dificulta a las personas para llegar al lugar, así mismo el sitio no cuenta con un parqueo para vehículos.

Con respecto a su página web presenta algunas debilidades, tales como que no cuenta con una versión traducida al idioma inglés, ya que muchas de las ayudas provienen del extranjero. También necesita un cifrado de SSL “candado de seguridad de la página“, ya que esto le genera seguridad a las personas que ingresan al sitio web, se debe verificar que los botones que redireccionan el sitio web hacia otros enlaces deben de estar en completo funcionamiento en este caso el botón que redirige a realizar donaciones. Por otra parte, el sitio web no cuenta con sistema automatizado para que las donaciones en dinero lleguen hacia una cuenta de la Fundación, por lo que se necesita una cuenta de un banco ligada a Paypal para que las personas dispuestas a donar con tan solo un click sin importar donde se encuentren estas brinden ayuda a través de donaciones monetarias. El sitio web no cuenta con un video institucional que incluya una entrevista de la fundadora de la institución, ya que a las personas esto les genera una mayor confianza y seguridad de conocer quien es la persona detrás de todo y quien va a recibir el dinero a como también quien lo va a administrar.

Por último, otra debilidad es la falta de una campaña de comunicación que tenga un objetivo, estrategia y táctica con unidad de contenido y mensaje que se adapte en distintos medios digitales como redes sociales, sitios web entre otros.

\section{b. Definición del problema}

Limitaciones que posee la Fundación Bandera Blanca para la captación de públicos extranjeros en edades de 25 a 35 años, capaces de realizar donaciones de dinero que permitan mantener en funcionamiento la Fundación, con el fin de brindar alimentación y apoyo académico a poblaciones vulnerables, durante el segundo cuatrimestre del 2019. 


\section{c. Planteamiento del problema}

¿Qué limitaciones posee la Fundación Bandera Blanca para la captación de públicos extranjeros que se encuentran en edades de 25 a 35 años, capaces de realizar donaciones de dinero que permitan mantener en funcionamiento la Fundación, con el fin de brindar alimentación y apoyo académico a poblaciones vulnerables, durante el segundo cuatrimestre del 2019?

\section{Metodología}

\section{a. Árbol de problema:}

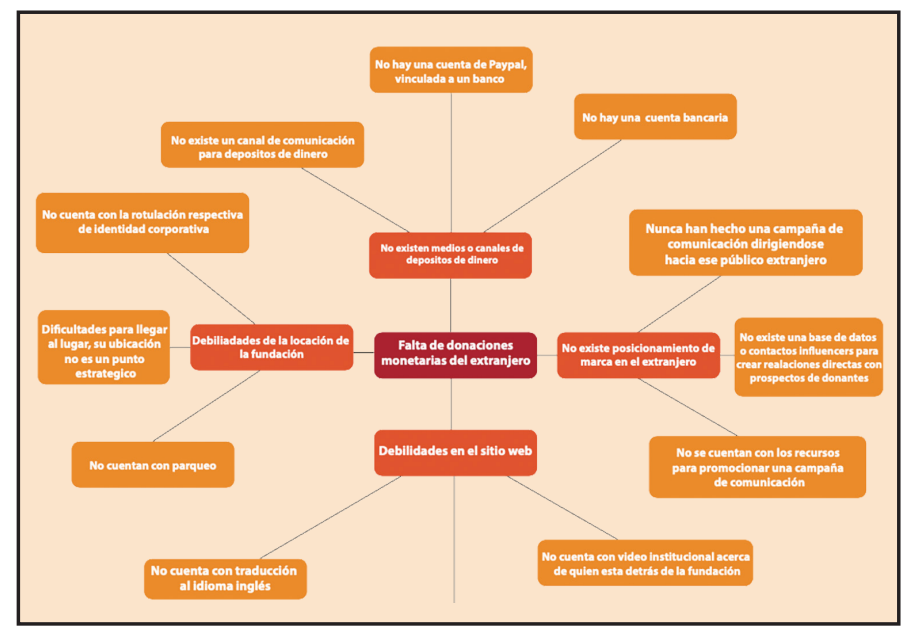

Fig. \#1. Árbol de problemas, elaboración del autor.

\section{b. Planteamiento de objetivo general y objetivos específicos (INVESTIGACIÓN)}

\section{Objetivo general}

Analizar las limitaciones que posee la Fundación Bandera Blanca para la captación de públicos extranjeros que se encuentran en edades de 25 a 35 años, capaces de realizar donaciones de dinero que permitan mantener en funcionamiento la Fundación, con el fin de brindar alimentación y apoyo académico a poblaciones vulnerables, durante el segundo cuatrimestre del 2019.

\section{Objetivos Específicos}

- Determinar el funcionamiento de diferentes herramientas automatizadas de fácil acceso para que prospectos donantes realicen sus ayudas monetarias de forma rápida $\mathrm{y}$ desde cualquier parte del mundo.

- Definir los diferentes canales o medios de comunicación en los cuales los prospectos donantes pasan su mayor tiempo.

- Señalar contactos y datos de prospectos de influenciadores para abarcar público en redes sociales de manera más directa y concisa, sin perder la misión y visión que posee la Fundación.

\section{c. Árbol de soluciones}

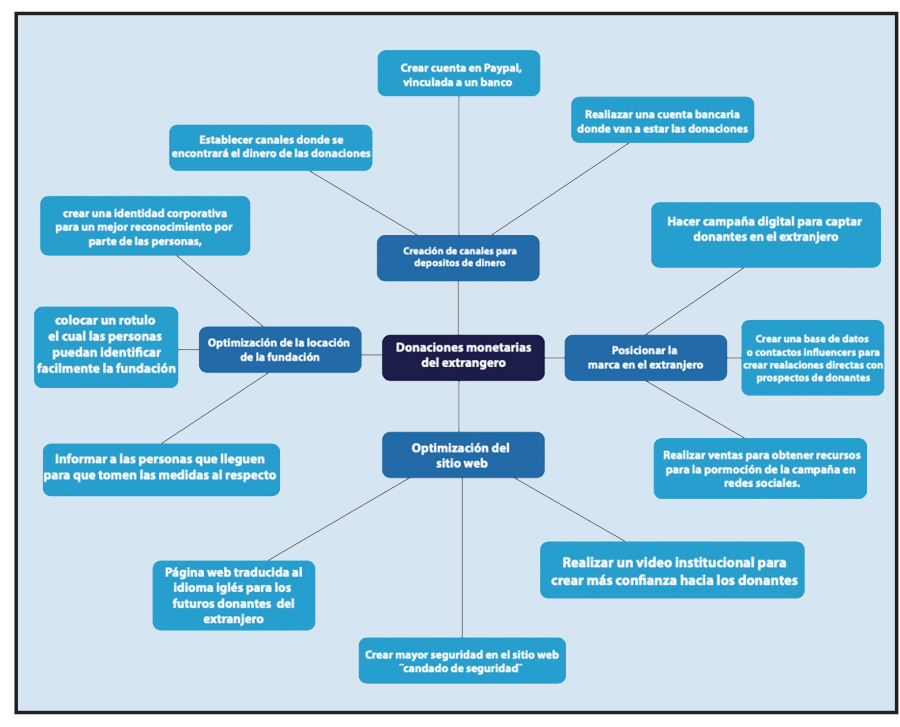

Fig. 2. Árbol de soluciones, elaborado por el autor.

\section{Desarrollo}

\section{a. Propuesta del Proyecto}

\section{Objetivo general}

Desarrollar diferentes alternativas y soluciones para la captación de públicos extranjeros que se encuentran en edades de 25 a 35 años, capaces de realizar donaciones de dinero a la Fundación Bandera Blanca, con el fin de brindar alimentación y apoyo académico a poblaciones vulnerables, durante el segundo cuatrimestre del 2019.

\section{Objetivos Específicos}

- Crear una nueva pestaña en la página web traducida al idioma inglés.

- Realizar una campaña para redes sociales enfocada al público extranjero.

- Determinar los lineamientos para ligar una cuenta de PayPal en Costa Rica para facilitar la recepción de donaciones.

- Crear una lista de posibles influenciadores que puedan ayudar a la organización.

\section{b. Ciclo de vida del proyecto}

\section{Evaluación inicial}

Para este proyecto, la etapa de evaluación inicial se realizó una llamada telefónica con la señora Berlín 
Castro, quien es la coordinadora de proyectos y principal funcionaria de la Fundación. Se entabló una conversación acerca del contexto actual de la Fundación y las necesidades que tienen en este momento. Berlín comentó que la Fundación pasa por un momento de ralentización económica al igual que todo el país. Por lo tanto, definió tres posibles grandes áreas en la que le gustaría la ayuda del equipo.

La primera tenía que ver con algún mecanismo de captar más donaciones, específicamente de personas en el extranjero. Ella quería que mediante alguna campaña o alguna posible solución digital; las personas en el extranjero se dieran cuenta de la Fundación. El segundo problema que tenía era que las personas voluntarias que llegaban no identificaban la Fundación. Para resolver esto, a ella le gustaría que el equipo propusiera alguna intervención a la fachada de los comedores, ya sea una nueva lona, un rótulo o un mural. Por último, la señora Castro comentó que otro de los retos era que los voluntarios no estaban muy claros con el tipo de trabajo y las situaciones a las que se iban a enfrentar en la Fundación. Por lo tanto, a ella le gustaría hacerle mejoras al proceso de inducción de voluntarios, ya sea mediante la creación de alguna publicación o solución digital que ayudará a cerrar esta brecha de desinformación.

Después de la conversación con Berlín, el equipo de trabajo se reunió para investigar en las redes sociales y la página de la Fundación. De esta manera se podía hacer una revisión cruzada de la información que la señora Castro había suministrado.

\section{Planificación}

Para el proceso de planificación, el equipo se reunió en la clase para discutir la información recolectada y llegar a un acuerdo de cómo se iba a proceder con el proyecto. Se comenzó con ejercicio de mesa redonda donde todos los integrantes del equipo daban su opinión acerca de cuál de todas las necesidades de la Fundación se iban a solucionar. Se tomó en cuenta la complejidad de cada una de las situaciones, los recursos y las restricciones físicas y temporales. Se llegó al acuerdo de que la situación de las donaciones por parte de extranjeros era el tema de mayor urgencia. Seguidamente, se dieron una serie de ejercicios con planos cartesianos y listas de prioridades para llegar a determinar la mejor manera de resolver el problema. Por último, los integrantes se dividieron en duplas de trabajo y se repartieron las tareas que había que realizar. Seguidamente se establecieron fechas de entrega para cada una de las tareas.

\section{Ejecución y seguimiento}

El proceso de ejecución del proyecto se realizó por duplas. Cada una decidió cómo y cada cuánto trabajar, con total autonomía siempre y cuando cumplieran con las fechas de entrega. Se utilizó la aplicación Bitrix24 como herramienta de organización para el proyecto. Esta permite a todo el equipo estar en constante comunicación, de igual manera sus herramientas de visibilidad de tareas permiten que haya completa transparencia entre los usuarios. Esto último facilitó los controles de seguimiento de las tareas. Para facilitar el proceso y no hacer chequeos innecesarios aparte de las fechas de entrega, se establecieron puntos de control en el calendario. Estos permiten a todo el equipo ver el estado de las tareas y la vez tienen suficiente tiempo antes de la fecha final de entrega en caso de que haya que tomar medidas de contención.

\section{Evaluación}

La clave del éxito de esta fase se basó en el correcto establecimiento de objetivos e indicadores de éxito. Esto facilitó en gran parte el proceso de evaluación puesto que había métricas a las cuales se podía consultar. De igual manera la satisfacción de los miembros de la Fundación es uno de los valores clave para determinar el grado de éxito dentro del proyecto. A esto se le debe agregar la función de la profesora Olga Marta Ramírez cuya supervisión a lo largo del proyecto y su función como ente evaluador en el lado académico, facilitó la demostración del impacto que puede llegar a tener el proyecto. Por último, se planea consultar con la señora Berlín Castro después de un tiempo prudencial de que se haya entregado el proyecto. Esto como mecanismo de retrospectiva y evaluación del proyecto.

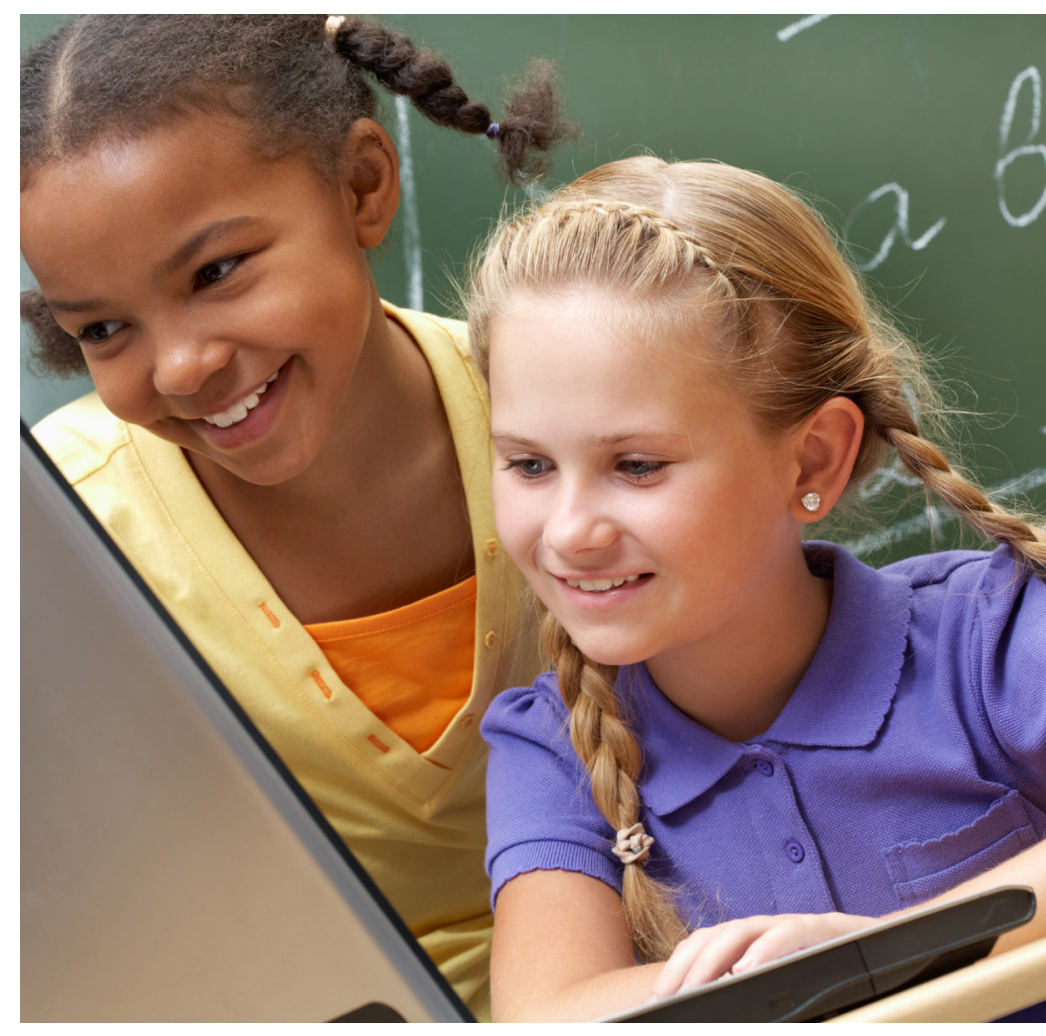




\section{c. Instrumentos de planificación y gestión}

\section{Creación de la Estructura de Descomposición de Trabajo (EDT)}

Tabla 1. Estructura de Descomposición de Trabajo

\begin{tabular}{|c|c|c|}
\hline Tarea & Duración & Responsable(s) \\
\hline Tener una computadora con acceso a internet y una cuenta de correo habilitada. & \multirow[t]{2}{*}{10 minutos } & \multirow[t]{2}{*}{ Administrador de proyectos } \\
\hline $\begin{array}{l}\text { Enviar un correo al cliente pidiéndole una reunión e intercambiar } \\
\text { teléfonos para discutir los alcances del proyecto y el contexto. }\end{array}$ & & \\
\hline Reunión con equipo de trabajo para distribuirse las tareas. & 3 horas & Todo el equipo \\
\hline Llamada al cliente de todo el equipo para discutir el proyecto. & 30 minutos & Todo el equipo \\
\hline Analizar la situación de la empresa y tomar decisiones. & 3 horas & Todo el equipo \\
\hline Una vez sacada las conclusiones, se pelotea una idea y concepto para la campaña. & 2 horas & Todo el equipo \\
\hline Se revisa si el cliente tiene cuenta en PayPal para donaciones del exterior. & 30 minutos & Equipo Estrella \\
\hline Se le pide a la cliente que comparta material vía correo electrónico. & 20 minutos & Administrador de proyectos \\
\hline Se revisa la posibilidad de usar influencer y se busca posibles colaboradores. & 2 horas & Equipo Influencer \\
\hline $\begin{array}{l}\text { Se contacta con el administrador de la web para usar los passwords } \\
\text { delsite y poder hacer la traducción de página. }\end{array}$ & 20 minutos & Equipo Site \\
\hline $\begin{array}{l}\text { Se revisan las redes sociales para fotografías y ver } \\
\text { si es necesario tomar fotos nuevas y se hacen propuestas de gráficas. }\end{array}$ & 2 horas & Equipo de campaña \\
\hline Se convoca una visita al establecimiento para una toma de fotos vía teléfono. & 30 minutos & Administrador de proyectos \\
\hline Se hace un diagrama fácil de entender para abrir una cuenta en PayPal y en Wink. & 3 horas & Equipo Estrella \\
\hline Una vez ya con los passwords del site se procede a editar el site. & 5 horas & Equipo Site \\
\hline $\begin{array}{l}\text { Se hace la visita al establecimiento para la toma de fotos y se muestran } \\
\text { las propuestas e idea para su aprobación. }\end{array}$ & 3 horas & Equipo de campaña \\
\hline Se hace el revelado digital de las fotos & 1 hora & Equipo de campaña \\
\hline Se escogen las fotos. & 30 minutos & Equipo de campaña \\
\hline Se editan y retocan las fotos escogidas. & 1 hora & Equipo de campaña \\
\hline Se montan las gráficas con las fotos finales. & 30 minutos & Equipo de campaña \\
\hline Se subtitula en inglés uno de los videos dados por el cliente. & 3 horas & Equipo de campaña \\
\hline Se hace render y se deja el video listo para subir a youtube & 5 minutos & Equipo de campaña \\
\hline Se monta el video en el site & 5 minutos & Equipo Site \\
\hline Una vez ya montadas las gráficas, video y el site, se hace un racional creativo & 30 minutos & Equipo de campaña \\
\hline Se monta la presentación con todos los materiales. & 2 horas & Equipo de campaña \\
\hline Se presenta el proyecto al cliente & 1 hora & Todo el equipo \\
\hline
\end{tabular}




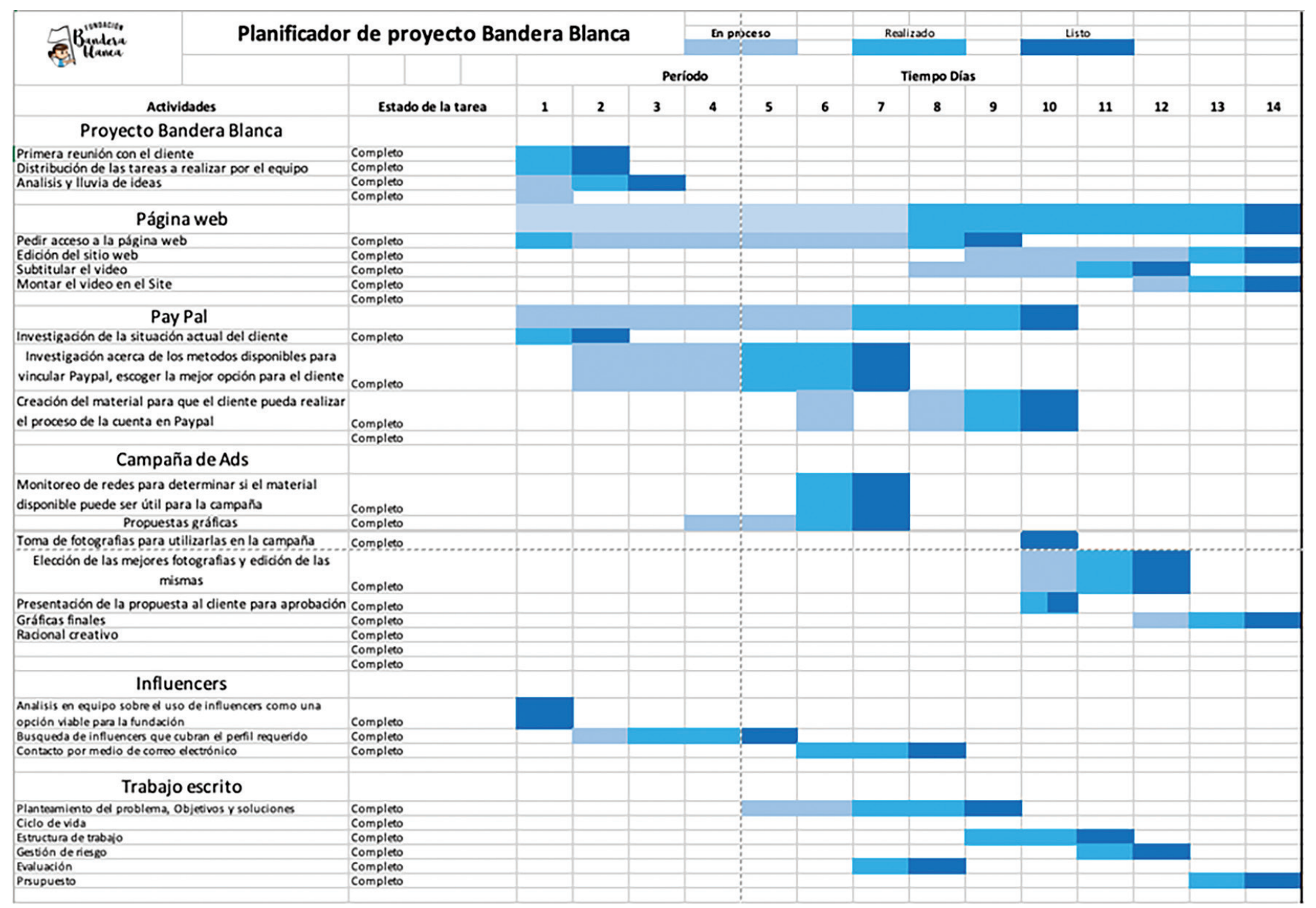

Figura 3. Planificación del proyecto, elaborado por el autor.

\section{d. Aspectos operativos y financieros del proyecto}

Los aspectos operativos y financieros del proyecto son de los ámbitos que se deben cuidar más, de ellos depende el éxito o fracaso de un proyecto, ya que es todo un ensamble coordinado. En el caso del proyecto de campaña digital para la Fundación Bandera Blanca, esta fue la división:

\section{Análisis del talento humano}

La gestión del talento humano es un proceso estratégico para retener a las personas que conforman equipos de alto rendimiento y, además, identificar potenciales talentos en las organizaciones.

En los proyectos, el capital humano es crítico para unificar todos los elementos y proveer una alta productividad en la implementación de sus tareas y actividades diarias. Para el proyecto de Fundación Bandera Blanca, el personal se dividió de la siguiente manera:

Web: Esta sección, conformada por Katherine y Michelle se encargaba de la programación y diseño web de las nuevas pestañas de la página: English y Donate. Estas dos pestañas eran el destino final de la campaña digital para atraer donantes extranjeros de habla inglesa, aquí las personas encontraban la información en inglés y el enlace directo a Paypal para hacer sus donaciones.

Redacción: La redacción estuvo a cargo de Alejandro y Katherine. Debido al target de la campaña, era necesario redactar copys e información en inglés.

Plataformas para transacciones monetarias: $\mathrm{La}$ investigación de las plataformas más viables para recibir las donaciones estuvo a cargo de Ronald y Estrellita, ellos se encargaron de sopesar la lista de pros y contras de las mismas y así elegir la mejor para el proyecto. Además, elaboraron una guía paso a paso para que la dueña de la fundación implementara la herramienta seleccionada.

Campaña digital: La campaña digital, dirigida principalmente con anuncios pautados, fue diseñada por Nahuel y Siloh, quiénes se encargaron de los artes para redes, la estrategia, el cronograma y el material fotográfico a utilizar en la campaña.

Búsqueda y contacto de influencers: El rubro de influencers fue asumido por Timothy, Alejandro 
y Siloh. Se encargaron de crear enlaces con los influencers que podrían eventualmente apoyar la fundación y lograr participación del público que los sigue. Estos tenían que tener una serie de características para ser incluidos dentro de la lista, por ejemplo, tener afinidad/o ser cristiano, mensaje positivo, que le gusten los niños, con público extranjero.

\section{e. Análisis del recurso físico}

El recurso físico hace referencia a todos los bienes tangibles, en poder de la empresa, que son susceptibles de ser utilizados para asegurar el normal desempeño de sus procesos operativos y cumplimiento de objetivos. Esta gestión comprende la adquisición, manejo y administración de equipos e inmuebles necesarios para la realización de las tareas y los procesos para su almacenamiento, distribución y mantenimiento.

Al contar con recursos monetarios nulos y poco tiempo disponible, el equipo de trabajo utilizó recursos propios (laptops, internet, programas de edición, etc.) para la realización del proyecto, y se realizó una reunión semanal para verificar los avances, que se habían previamente agendado en la herramienta Bitrix24.

\section{f. Gestión del tiempo}

Todo proyecto obligatoriamente se encuentra ligado a varias fechas de entrega que hay que cumplir para poder ejecutar lo inicialmente propuesto. Por esta razón, se planea cada una de las actividades y se distribuye el tiempo de manera eficaz, teniendo en cuenta cualquier imprevisto que pueda surgir.

El proyecto de Bandera Blanca tenía como fecha límite el 31 de agosto para entregar la campaña al cliente, sin embargo, la presentación del proyecto al cliente debía ser el 28 de agosto. Por lo tanto, la distribución del trabajo fue la siguiente:

Tabla 2. Distribución de Trabajo

\begin{tabular}{l|l}
\hline Actividad & Fecha \\
\hline Primera reunión como grupo & 7 de agosto \\
\hline Primer avance & 21 de agosto \\
\hline Trabajo escrito & 27 de agosto \\
\hline Página web & 27 de agosto \\
\hline Presentación Ejecutiva & 27 de agosto \\
\hline Exposición con el cliente & 28 de agosto \\
\hline Tiempo final para envío de material & 30 de agosto \\
\hline
\end{tabular}

\section{Resultados}

Los estudiantes no tienen problemas en la creación de sus cuentas de acceso a la plataforma virtual. El 100\% de los participantes crearon sus cuentas en la clase siguiendo las instrucciones del docente, en el primer periodo de estudio tres estudiantes requirieron asistencia entre las clases de semana uno y dos para acceder nuevamente al sistema, luego de interactuar con los estudiantes se determinó que se debió a restricciones de acceso en los lugares de trabajo. En el segundo periodo, dos estudiantes que iniciaron clases la segunda semana del periodo lectivo requirieron material adicional.

\section{a. Gestión del riesgo}

La gestión del riesgo se realiza con el fin de identificar los riesgos que se puedan presentar durante el proyecto, planificar la respuesta y hacer el seguimiento necesario para aumentar la probabilidad e impacto de los eventos positivos y disminuir los eventos adversos.

En este proyecto, el tiempo y el aspecto financiero eran los principales factores de riesgo, tal como los muestra el siguiente cuadro:

Tabla 3. Análisis de Riesgo.

\begin{tabular}{l|l|l}
\hline Evento & Descripción del riesgo & $\begin{array}{l}\text { Nivel de } \\
\text { amenaza }\end{array}$ \\
\hline Financiero & $\begin{array}{l}\text { Escaso presupuesto para } \\
\text { pauta de la campaña digital }\end{array}$ & Medio \\
\hline Tiempo & No se puede entregar a tiempo & Alto \\
\hline Humano & $\begin{array}{l}\text { Algún miembro del equipo } \\
\text { se enferma }\end{array}$ & Medio \\
\hline
\end{tabular}

\section{Discusión de resultados}

\section{a. Evaluación de proyectos (PROPUESTA)}

\section{Indicadores de evaluación.}

Al ser este un proyecto caracterizado por llevarse a cabo en un lapso de tiempo bastante reducido, las mediciones tendrán a variar un poco, sin embargo, podemos rescatar varios indicadores de evaluación que nos permiten ver que el proyecto fue exitoso en base a los objetivos trazados.

Primeramente, el hecho de que el cliente haya estado a gusto con la idea presentada por el equipo de trabajo para el proyecto, con la manera en que se le indicó se iba a llevar cabo el mismo, y así con esto lo haya aprobado, es un indicador de que se iba por buen camino, y desde ya se estaba cumpliendo con las expectativas de los miembros de la fundación. 
Otro de los objetivos establecidos al inicio de la campaña buscaba agregar un segundo idioma (inglés) a la página web de la fundación que le permitiera ser comprendida por personas del extranjero.

El hacer entrega a las encargadas de la Fundación Bandera Blanca una versión actualizada de su página web, con la respectiva implementación de un segundo idioma, es un indicador de que el proyecto fue exitoso, no solo porque cumple con las necesidades del cliente, sino, porque también cumple el objetivo establecido previamente por el equipo de trabajo.

Mediciones finales o más profundas en torno al impacto que tuvo la campaña digital como tal en su público meta, desde que fue lanzada hasta su finalización, será un poco más complejo de identificar por el momento por motivos de tiempo, principalmente porque será en esta etapa donde el propio cliente será el encargado de velar por darle seguimiento, sin embargo, no se descarta realizar un acercamiento próximamente que permita denotar resultados más específicos del proyecto realizado.

\section{b. Programas de Gestión de Proyectos Aplicación usada para la gestión del proyecto}

Para gestionar este proyecto se escogió la aplicación digital Bitrix24. Las principales razones por las cuales se escogió esta alternativa y no otras fueron las siguientes:

- Es una aplicación de libre uso que no requiere inversión monetaria en su versión básica. Esto fue un factor determinante puesto que la fundación y el equipo de trabajo tienen recursos económicos limitados.

- Permite crear espacios de trabajo de hasta quince personas, un número adecuado para la cantidad de colaboradores involucrados en el proyecto.

- Posee una gran cantidad de herramientas que facilitan la administración de un proyecto, como por ejemplo la posibilidad de crear gráficos de Gantt, organigramas y chat de comunicación general.

- Posee cualidades responsive lo que le permite ser accesada desde una página web o un dispositivo móvil, incluso tiene su propia aplicación.

- $\mathrm{Su}$ interfaz se asemeja a la de una red social, lo que facilita su adopción de uso por parte de nuevos miembros.

A continuación, una serie de imágenes retratando el uso de la aplicación por parte del equipo:

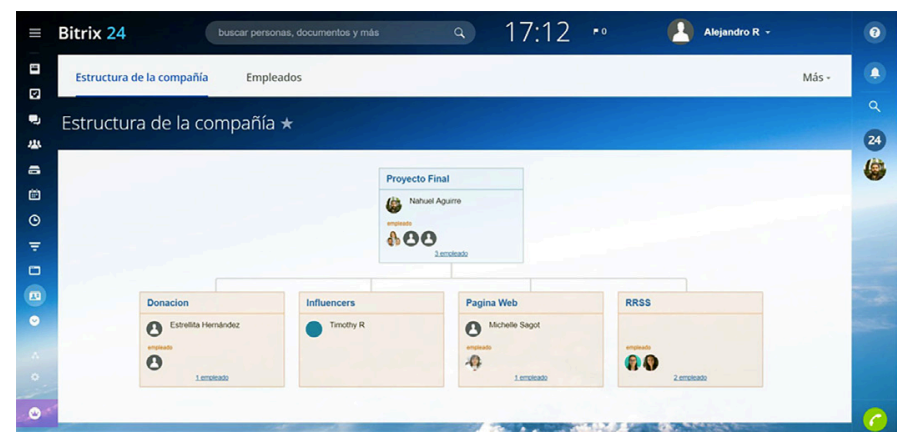

Figura 4. Captura de pantalla de la página de ingreso a la aplicación, elaboración propia.

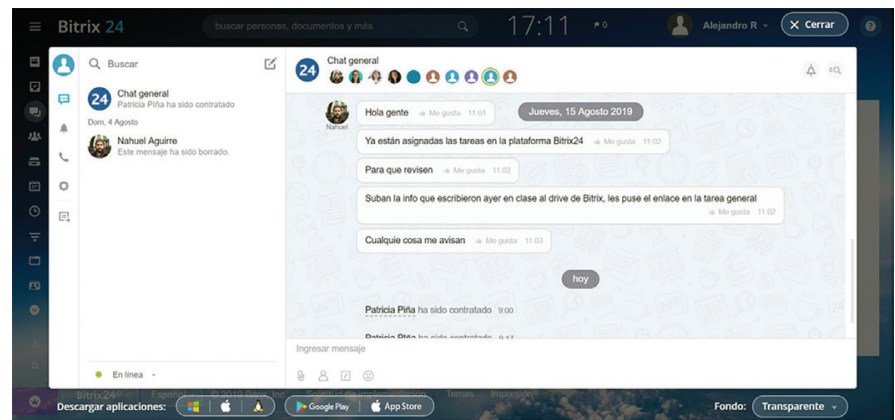

Figura 4. Captura de pantalla de la página de ingreso a la aplicación, elaboración propia.

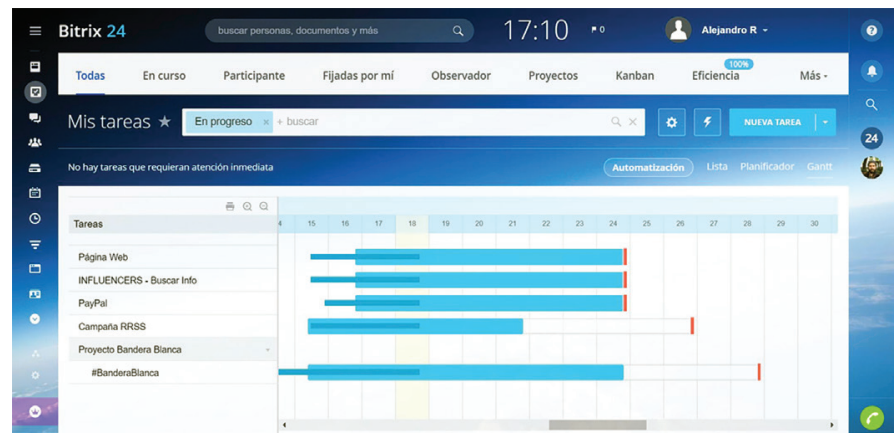

Figura 5. Control de la planificación, elaborado por el autor.

\section{c. Presupuesto}

Al ser una organización sin fines de lucro y con tanto problema para recolectar dinero y mantener el proyecto a flote, se decidió no cobrar costos operativos ni usar el pensamiento de cobrar lo que vale el trabajo de cada uno, ni gastos en general. Al final solo cobrar por horas persona como parte de la ayuda a la organización. Es decir, un cálculo de un salario de C.1.000.000 mensual y se dividió en 4 semanas, que es el promedio que tiene un mes y ese total es de 50.000 colones por día y después se dividió en las 8 horas diarias para un total final de C.6.250 colones por hora.

En todo el proyecto se invirtieron un total de 98 horas tanto de manera individual como en equipo y el precio final fue de C.612.500 colones sin IVA incluido. 
A este resultado se debe agregar una donación en efectivo realizada para la Fundación Bandera Blanca de C.10 000 colones por parte del equipo de trabajo.

A continuación, se presenta una serie de capturas de pantalla de los productos.

\section{Página web}

La página web es responsive y navegable tanto en móvil como en computador. Aquí algunos screenshots:

\section{Página English}

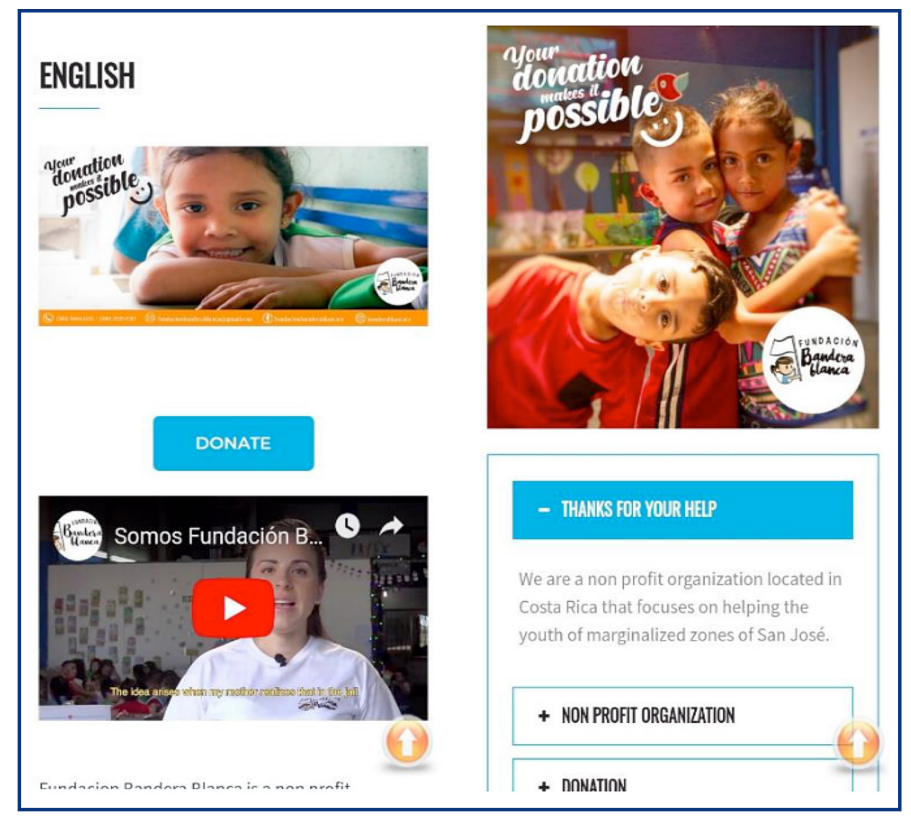

Figura 6. Captura de pantallas de la aplicación en inglés, elaboración propia.

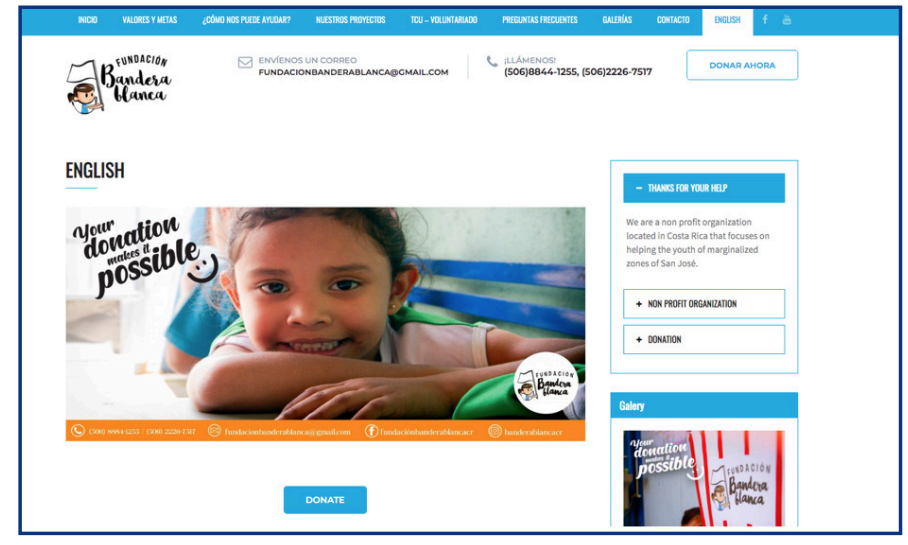

Figura 7. Página en inglés de la aplicación.

\section{Agradecimientos}

A los estudiantes participantes:

Katherine Agüero | Nahuel Aguirre | Estrellita Hernández | Siloh Piña | Timothy Ryan | Alejandro Rojas | Michelle Sagot | Ronald Solís

\section{Referencias}

[1] Triana M (s.f) El Constructivismo: Paradigma de la Escuela Contemporánea.

[2] Cárdenas Páez A. (2011) Piaget: lenguaje, conocimiento y educación Revista Colombianade Educación, N. ${ }^{\circ} 60$. Primer semestre, Bogotá.

[3] Castro B. Entrevista personal, setiembre 2019 